\title{
Physiologic effects of GHRH in patients with HIV lipodystrophy: a model of acquired visceral adiposity
}

\section{Steven Grinspoon}

HIV-infected patients using highly active antiretroviral therapy show progressive changes in fat distribution; a large percentage of patients accumulate excess visceral fat, which might contribute to increased cardiovascular risk in this group. Recent data show reduced growth hormone secretion-specifically decreased growth hormone pulse amplitude and overall night-time secretion-in association with excess visceral fat in this population.

Previous studies into the use of growth hormone in HIV-infected patients with abdominal fat accumulation have mostly used supraphysiologic doses; however, two studies (Koutkia $\mathrm{P}$ et al. [2004] JAMA 292: 210-218; Falutz J et al. [2005] AIDS 19: 1279-1287) suggest the use of a novel, more physiologic strategy. This strategy involves using growth-hormone-releasing hormone (GHRH) to augment growth hormone secretion and, therefore, reduce abdominal fat accumulation. In the first study, an analog that contained amino acids 1-29 of GHRH resulted in a significant reduction in truncal fat and a significant redistribution of fat away from the trunk, toward the extremities - a pattern associated with lower cardiovascular risk. In contrast to studies with high-dose growth hormone, there was no increase in glucose levels. In the second study, which used a longer-acting synthetic analog called GRF (1-44) (amino acids 1-44 of GHRH plus a hexonyl moiety at the $\mathrm{N}$ terminus), significant reductions in truncal fat and an improvement in triglyceride levels and the cholesterol: HDL-cholesterol ratio were also seen, again without deterioration in glucose levels, even when patients with diabetes were included. Both studies showed significant, but physiologic, increases in levels of insulin-like growth factor 1 .

The use of GHRH analogs has been considered previously for short stature and aging, two conditions characterized by relative reductions in growth hormone secretion. Use of $\mathrm{GHRH}$
It is reasonable

to ask

whether such

a treatment

could be used

among non-

HIV-infected

patients with

generalized

obesity

$S$ Grinspoon is an

Associate Professor

of Medicine,

Massachusetts General

Hospital and Harvard

Medical School,

Boston, MA, USA.

\section{Competing interests}

The author has received

consulting fees and

research support from

Theratechnologies Inc. and

lecture fees from Serono Inc.

www.nature.com/clinicalpractice doi:10.1038/ncpendmet0225 would not be appropriate in patients with true pituitary disease (e.g. that caused by a tumor or radiation), or with an inability to increase growth hormone in response to stimulation by a hypothalamic peptide. In contrast, in obesity, growth hormone levels are reduced, but preliminary data suggest this is a partial or relative reduction, and the capacity to respond to stimulation with GHRH is, therefore, probably intact-as found in the two studies on HIV lipodystrophy-among patients with severe abdominal fat accumulation.

Further long-term studies are necessary to determine the safety and efficacy of GHRH in viscerally obese patients. A number of important questions about GHRH remain to be answered, including those concerned with the net effect on cardiovascular risk, the duration of this effect, the optimal dose, and the longterm safety. One potential benefit of GHRH, in contrast to growth hormone, is that feedback inhibition is intact, potentially preventing supraphysiologic growth hormone secretion. Indeed, several studies have now been completed using GRF (1-44), which have proven its safety, even among patients with diabetes, and shown that dose titration is not necessary, as feedback of increased levels of insulin-like growth factor 1 on endogenous growth hormone secretion might accomplish the same purpose.

How might GHRH be used in the future? If it proves useful in reducing visceral fat in HIV lipodystrophy, it is reasonable to ask whether such a treatment could be used among nonHIV-infected patients with generalized obesity. Very few therapies have proven useful to decrease visceral fat in such patients. Levels of growth hormone appear reduced in obesity, and this might further contribute to accumulation of excess visceral fat in a vicious cycle. Physiologic augmentation with a growth hormone secretagogue could potentially break this cycle, and prove useful as a novel strategy to reduce abdominal fat. 